\title{
Genome-Wide Expression Profiles Drive Discovery of Novel Compounds that Reduce Binge Drinking in Mice
}

\author{
Laura B Ferguson 1,5, Angela R Ozburn ${ }^{2,3,5}$, Igor Ponomarev', Pamela Metten ${ }^{2,3}$, Matthew Reilly \\ John C Crabbe ${ }^{2,3}$, R Adron Harris' and R Dayne Mayfield ${ }^{*, 1}$ \\ 'The Waggoner Center for Alcohol and Addiction Research, The University of Texas at Austin, Austin, TX, USA; ${ }^{2}$ Department of Behavioral \\ Neuroscience, Oregon Health and Science University, Portland, OR, USA; ${ }^{3}$ VA Portland Health Care System, Portland, OR, USA; ${ }^{4}$ Division of \\ Neuroscience and Behavior, National Institute on Alcohol Abuse and Alcoholism, Bethesda, MD, USA
}

\begin{abstract}
Transcriptome-based drug discovery has identified new treatments for some complex diseases, but has not been applied to alcohol use disorder (AUD) or other psychiatric diseases, where there is a critical need for improved pharmacotherapies. High Drinking in the Dark (HDID-I) mice are a genetic model of AUD risk that have been selectively bred (from the HS/Npt line) to achieve intoxicating blood alcohol levels (BALs) after binge-like drinking. We compared brain gene expression of HDID-I and HS/Npt mice, to determine a molecular signature for genetic risk for high intensity, binge-like drinking. Using multiple computational methods, we queried LINCS-L I000 (Library of Integrated Network-Based Cellular Signatures), a database containing gene expression signatures of thousands of compounds, to predict candidate drugs with the greatest potential to decrease alcohol consumption. Our analyses predicted novel compounds for testing, many with anti-inflammatory properties, providing further support for a neuroimmune mechanism of excessive alcohol drinking. We validated the top 2 candidates in vivo as a proof-of-concept. Terreic acid (a Bruton's tyrosine kinase inhibitor) and pergolide (a dopamine and serotonin receptor agonist) robustly reduced alcohol intake and BALs in HDID-I mice, providing the first evidence for transcriptome-based drug discovery to target an addiction trait. Effective drug treatments for many psychiatric diseases are lacking, and the emerging tools and approaches outlined here offer researchers studying complex diseases renewed opportunities to discover new or repurpose existing compounds and expedite treatment options.

Neuropsychopharmacology (2018) 43, 1257-1266; doi:I0.1038/npp.2017.30।; published online I4 March 2018
\end{abstract}

\section{INTRODUCTION}

Brain transcriptome data are becoming more affordable to generate and more accessible via large public repositories. The healthcare field stands to benefit tremendously by harnessing the power of high-throughput omics data (Chen and Butte, 2016). One approach is to compare the molecular profiles disrupted by disease states with those altered by therapeutic compounds to target the disease phenotype (Lamb et al, 2006; Nakagawa et al, 2016; Wagner et al, 2015). It is now feasible to integrate the molecular profiles of drugs and diseases to advance drug discovery and repurposing due to increased access to transcriptome data from public repositories and the continued development of databases that catalog the transcriptional response to different pharmacological agents in human cell lines, such as the Broad Institute's Connectivity Map database (CMap; https://www. broadinstitute.org/connectivity-map-cmap) and the Library

*Correspondence: Dr RD Mayfield, Waggoner Center for Alcohol and Addiction Research, The University of Texas at Austin, I University Station, A4800, Austin, TX 787I2, USA, Tel: +5I2 232-7578,

Fax: + 512232 2525, E-mail: dayne.mayfield@austin.utexas.edu

${ }^{5}$ These authors contributed equally to this work.

Received 12 July 2017; revised 6 November 2017; accepted 24 November 2017; accepted article preview online 18 December 2017 of Integrated Network-Based Cellular Signatures (LINCSL1000; http://www.lincsproject.org/; http://clue.io). This approach has successfully pointed to novel therapeutics for several diseases, mostly cancers, as the cancer research field has devoted considerable effort to using genomic data for drug discovery, but also for other diseases such as inflammatory bowel disease and skeletal muscle atrophy (see Chen and Butte, 2016; Iorio et al, 2013 for review). There are limited studies applying CMap to neurological diseases including Huntington's (Smalley et al, 2016), Parkinson's (Gao et al, 2014), and Alzheimer's (Fowler et al, 2015). One study used genome-wide association study data for psychiatric illnesses to query CMap and identified potential therapeutic compounds, but did not provide any validation (So et al, 2017). The number of cell lines and compounds in the CMap database was recently expanded significantly through LINCS-L1000. This expansion relied on the development of the L1000 technology, in which the effects of a drug on expression of $\sim 22000$ genes were inferred from the direct measurement of 1000 'landmark' genes (Subramanian et al, 2017). LINCS-L1000 has been used for drug discovery, albeit to a much lesser extent than CMap (Lipponen et al, 2016; Liu et al, 2015; Mirza et al, 2017; Siavelis et al, 2016). One new LINCS-L1000 study evaluated a modification of the computational approach to 
select and prioritize compounds for breast, colon, and liver cancer, providing in vivo validation for the top compound and found a positive correlation between a drug's negative connectivity score with their cancer signature and its halfmaximal inhibitory concentration, a measure of in vitro drug efficacy (Chen et al, 2017). LINCS-L1000 is therefore emerging as a vital tool for drug discovery and repurposing, but its predictive power for psychiatric disorders, where there is a large unmet need for improved pharmacotherapeutics, has not been evaluated.

Alcohol use disorder (AUD) is a devastating psychiatric illness with few efficacious therapies (Koob and Mason, 2016). A major risk factor for developing AUD is binge drinking, a drinking pattern that raises blood alcohol levels (BALs) to those that significantly impair motor and cognitive functioning. Binge drinking carries a high cost to society and the individual in the form of AUD risk, vehicular accidents, violence, injury, sexual assault, and death (Esser et al, 2014).

Pre-clinical models play a central role in the target identification and validation process. Here we studied High Drinking in the Dark (HDID-1) mice, a genetic model of high intensity, binge-like drinking, as a predictive model for drug discovery and repurposing (Crabbe et al, 2009). Gene expression data sets from experimentally naive HDID-1 and $\mathrm{HS} / \mathrm{Npt}$ (the genetically diverse, low-drinking line from which HDID-1 were selectively bred) were used to construct transcriptional signatures from eight brain regions known to be important for alcohol drinking (Ozburn et al, 2015). The HDID-1 signatures, reflecting the molecular state induced by selection for drinking to intoxicating BALs, were submitted as a LINCS-L1000 query to predict drugs with the potential to reduce binge-like drinking in HDID-1 mice. The rationale for our application of this computational approach is that genetic selection has altered brain networks and these alterations are reflected in changes in gene expression. The basis of this drug selection algorithm is that the drug signature can be compared with the disease signature to select compounds that will 'correct' the biological functions of the system. A LINCS-L1000 query is comprised of three steps: (1) construct the input signature, (2) run the comparison algorithm, and (3) prioritize the candidate compounds for in vivo testing. At each step, there are several user-defined parameters that can greatly affect the outcome of a query, although these have not been carefully investigated. We reasoned that a strong candidate compound should not depend heavily on methodological choices. Thus, we took a combinatorial approach and ran several LINCS-L1000 queries with varying parameter settings, finally prioritizing drug candidates that scored highly across all analyses. We exploited the availability of multiple gene expression data sets to conduct a meta-analysis, which helped to mitigate technological and biological biases. Our proof-of-concept study successfully identified novel compounds and the top 2 candidates tested in vivo significantly reduced alcohol intake and BALs in HDID-1 mice.

\section{MATERIALS AND METHODS}

\section{Animals}

Mice were bred and housed in the Veterinary Medical Unit at the Veterans Affairs Portland Health Care System
(Portland, OR). Generation and selective breeding information for these mice were described previously (Crabbe et al, 2014). Briefly, selective breeding began with $\mathrm{HS} / \mathrm{Npt}$ mice (an outbred, heterogeneous stock originally constructed by intercrossing eight inbred mouse strains (Crabbe et al, 2014; Crabbe et al, 2009). Mice attaining the highest BALs (after limited access to a $20 \%$ ethanol solution during their circadian dark cycle) were bred together each generation to increase the population's BALs. Experimentally naive adult male HDID-1 mice from selection generation 16 (S16) and $\mathrm{HS} / \mathrm{Npt}$ mice from unselected generation 68 (G68) (12 per genotype) were used for gene expression analysis. Experimentally naive adult male and female HDID-1 mice were used for in vivo drug testing (from generations S34-S36). Mice were bred and maintained on a reverse light cycle (lights off at $0930 \mathrm{~h}$, lights on at $2130 \mathrm{~h}$ ), with temperature held near $20^{\circ} \mathrm{C}$. Food (and water, except where noted) were available ad libitum. Mice were habituated to the behavioral testing room, individual housing, and new sippers/drinking tubes 5-7 days before experiment. All procedures were conducted in accordance with the NIH Guidelines for the Care and Use of Laboratory Animals and were approved by the local Institutional Animal Care and Use Committee.

\section{Processing and Analysis of Gene Expression Datasets}

We used laser capture microdissection to precisely collect tissue from the prefrontal cortex (PFC), nucleus accumbens core (AcbC), nucleus accumbens shell (AcbSh), bed nucleus of the stria terminalis (BNST), basolateral amygdala (BLA), central nucleus of the amygdala (CeA), and ventral tegmental area (VTA) of naive HDID-1 (S16) and HS/Npt lines (G68) (GEO accession number GSE93311). Iancu et al (2013) previously dissected the ventral striatum (VS) from naive HDID-1 (S22) and HS/Npt mice (G72) (48 mice/genotype) (GEO accession number GSE93515). Methods for the VS gene expression data set are described in detail elsewhere (Iancu et al, 2013). For the other seven brain areas, detailed methods are in Supplementary Materials and Methods. Briefly, total RNA was extracted, purified, amplified, and hybridized to Illumina Mouse WG-6 v2.0 Expression BeadChips (Illumina, Inc., San Diego, CA, USA). The data were preprocessed using the Bioconductor lumi package in the $\mathrm{R}$ programming environment ( $\mathrm{Du}$ et al, 2008). Differential expression analysis was conducted within each brain region using empirical Bayes moderated $t$-statistics from the Bioconductor limma package in $\mathrm{R}$ to compare $\mathrm{HS} / \mathrm{Npt}$ and HDID-1 mice (Ritchie et al, 2015).

\section{LINCS-L1000 Search}

We identified candidate compounds by submitting a LINCSL1000 query via C3 (Compute Connectivity on the Cloud), a command line interface with the LINCS-L1000 database. The LINCS-L1000 datasets can also be downloaded (GEO accession numbers GSE70138 and GSE92742). There are several user-defined parameters that affect the outcome of a query: the input signature, rowspace, and columnspace (discussed below). As the settings that produce the most biologically relevant outputs are not known a priori, we elected to run 32 LINCS-L1000 queries with different parameter settings and prioritize drug candidates that scored 
highly across all analyses. A LINCS-L1000 input signature is comprised an 'up' and a 'down' list of genes, and the top 100 upregulated genes and the top 100 downregulated genes for each brain region (HDID-1 vs HS/Npt mice) were used as the input signature in the current study (Supplementary Table S1). This represented the top $\sim 10 \%$ genes differentially expressed between HDID and HS/Npt mice $(p<0.05): 624$ in PFC, 1350 in AcbC, 1219 in AcbSh, 1666 in BNST, 1275 in BLA, 1238 in CeA, 1312 in VTA, and 1430 in VS. In addition, we generated a second input signature for each of the eight data sets comprised exclusively of the differentially expressed 'landmark' genes (Supplementary Table S1). Landmark genes are those that are directly measured to generate the L1000 gene expression database. As the L1000 platform is designed to imitate the Affymetrix HG-133A probe sets, we converted gene names to the corresponding HG-133A probe set using the 'hgu133a.db' package in $\mathrm{R}$ (Carlson, 2016). We ran the query in two gene rowspaces: the landmark and best inferred gene ('bing') rowspaces. The rowspace is the subset of genes within the LINCS-L1000 database used in the query to compute the connectivity scores. The 'bing' rowspace contains genes deemed by the Broad Institute to be the best inferred from the directly measured landmark genes. Therefore, we created two input signatures for each of the eight data sets and ran the query in two gene rowspaces $(2 \times 8 \times 2=32$ inputs $)$.

The LINCS-L1000 database was then queried with the 32 input signatures, and a connectivity score based on the Kolmogorov-Smirnov statistic was computed to assess the overlap of the input signature and more than 3000 compound's signatures in the LINCS-L1000 database (see Lamb et al, 2006 for details). A compound's connectivity scores range from -100 to +100 , where a negative score indicates dissimilarity between the HDID vs HS/Npt input and compound's signature, and a positive connectivity score indicates similarity. A connectivity score of 0 is assigned if there is no clear pattern in gene regulation between the input query and drug signature. We performed the queries in the 'touchstone' columnspace, which contains the most reproducible signatures for well-characterized compounds. We used the 'sig_gutc' tool available on C3 to summarize the connectivity scores and provide a measure of reliability. Each compound has been profiled under multiple experimental conditions (different cell lines, drug doses, and exposure time points). To attain a compound-level analysis, sig_gutc reports the maximum quartile connectivity score to summarize the scores across cell line, dose, and time point. The 25th and 75th percentile scores are computed for the distribution of scores for a compound across all experiments. The maximum quartile score is either the 25th or 75th percentile of a given set of connectivity scores, whichever magnitude is largest. The tool then ranks the connectivity score between the query signature and the compound signature, based upon the compound's pre-computed distribution of connectivity scores with the other hundreds of thousands of signatures in the LINCS-L1000 database. This provides a measure of the likelihood of a connectivity score for a drug given that drug's connectivity with the database as a whole, thus mitigating false positives from drugs with widespread effects on transcription.
Drugs

Terreic acid (catalog number SML0480, Sigma-Aldrich, St. Louis, MO, USA) was dissolved in saline and administered intraperitoneally at doses of $0,3.75,5,7.5$, or $15 \mathrm{mg} / \mathrm{kg}$. These doses have been shown to have anti-inflammatory, antimicrobial, and anti-tumor actions (Han et al, 2010; Kawakami et al, 1999; Kawakami et al, 2007; Olesen et al, 2014; Yamamoto et al, 1980). Pergolide mesylate salt (catalog number P8828, Sigma-Aldrich) was dissolved in saline and administered intraperitoneally at doses of $0,1,2$, or $4 \mathrm{mg} / \mathrm{kg}$. These doses have been shown to have anti-inflammatory actions, without altering locomotor activity or prepulse inhibition of startle in mice (Bendele et al, 1991; Martin and Bendesky, 1984; RalphWilliams et al, 2003). Injection volumes were $10 \mathrm{ml} / \mathrm{kg}$. A $20 \%$ ethanol solution ( $\mathrm{v} / \mathrm{v}$, in tap water) was used for the alcohol drinking experiments (catalog number 2701, Decon Labs) and an $8.5 \mathrm{mM}$ saccharin solution (in tap water) was used for the tastant drinking experiments (catalog number 24,431-7, SigmaAldrich). All solutions were prepared fresh daily.

\section{Drinking in the Dark}

Drinking in the dark (DID) was performed as described (Crabbe et al, 2014) with minor modifications. We performed 3 weeks of serial testing to examine the effects of candidate drugs on intake of $20 \%$ ethanol (week 1), water (week 2), or $8.5 \mathrm{mM}$ saccharin (week 3). These limited-access drinking sessions (termed DID sessions) were carried out on the first 2 days of each week. On the first DID day, $3 \mathrm{~h}$ after lights off, individual water bottles were replaced with a single graduated tube offering $20 \%$ ethanol in tap water $(\mathrm{v} / \mathrm{v})$ for $2 \mathrm{~h}$. At the end of the drinking session, the volume was recorded and water bottles were returned. On the second DID day, mice received an intraperitoneal injection of vehicle or drug $30 \mathrm{~min}$ before the DID session. For the second DID session, tube volumes were measured at $2 \mathrm{~h}$ (for terreic acid testing) or at 2 and $4 \mathrm{~h}$ (for pergolide testing). Terreic acid has a short duration of action based on our time course study (Supplementary Fig 2) and previous reports (Kawakami et al, 1999; Yamamoto et al, 1980). After the final volume of ethanol solution was recorded, a peri-orbital sinus blood sample $(20 \mu \mathrm{l})$ was collected to measure BALs (Finn et al, 2007). Mice were left undisturbed for the next 5 days. The second and third weeks of DID testing were carried out in the same manner, with the exception that water intake and then saccharin intake was measured and no blood samples were collected. An additional point on serial testing in mice from the terreic acid studies is discussed here. As the lowest dose of terreic acid tested $(3.75 \mathrm{mg} / \mathrm{kg})$ did not reduce ethanol drinking or BAL in week 1 of testing (Supplementary Fig 3a,b), we proceeded to test whether $5 \mathrm{mg} / \mathrm{kg}$ terreic acid would reduce ethanol drinking (as compared with vehicle) in week 2 . Thus, the schedule for this subgroup is shifted by one week (with water intake tested in week 3 and saccharin intake tested in week 4). Mice in this subgroup were either tested with vehicle four times or terreic acid four times before drinking assays. Pharmacological testing was carried out in two cohorts for each compound, where the number of mice tested for terreic acid was 23-24 mice/sex in the $0 \mathrm{mg} / \mathrm{kg}$ group, $12 \mathrm{mice} / \mathrm{sex}$ in the 3.75 and $5 \mathrm{mg} / \mathrm{kg}$ group, $12 \mathrm{mice} / \mathrm{sex}$ in the $7.5 \mathrm{mg} / \mathrm{kg}$ group, and $12 \mathrm{mice} / \mathrm{sex}$ in the 
$15 \mathrm{mg} / \mathrm{kg}$ group and the number of mice tested for pergolide was $23-24 \mathrm{mice} / \mathrm{sex}$ in the $0 \mathrm{mg} / \mathrm{kg}$ group, $12 \mathrm{mice} / \mathrm{sex}$ in the $1 \mathrm{mg} / \mathrm{kg}$ group, $12 \mathrm{mice} / \mathrm{sex}$ in the $2 \mathrm{mg} / \mathrm{kg}$ group, and 12 $\mathrm{mice} / \mathrm{sex}$ in the $4 \mathrm{mg} / \mathrm{kg}$ group.

\section{Locomotor Activity Assay}

To ascertain whether reductions in ethanol drinking could be explained by general sedation (reduced locomotor activity), we assessed locomotor activity in response to vehicle or drug (terreic acid or pergolide) by testing a subset of mice from the serial DID testing for each drug. For terreic acid, mice had previously been treated with either $0,3.75$, or $5 \mathrm{mg} / \mathrm{kg}$ prior to DID (for a total of four previous exposures to either vehicle or terreic acid). For pergolide, mice had previously been treated with either 0 or $1 \mathrm{mg} / \mathrm{kg}$ pergolide before DID (for a total of three previous exposures to pergolide). Unlike serial testing before DID for the different fluids, seven to eight mice/sex/dose were randomized into new treatment groups for activity testing.

At $2.5 \mathrm{~h}$ after lights out, mice received an intraperitoneal injection of vehicle or a dose of a drug. For terreic acid, mice received 0,5 , or $7.5 \mathrm{mg} / \mathrm{kg}$. For pergolide, mice received 0 , 1 , or $2 \mathrm{mg} / \mathrm{kg}$. Three hours after lights out, mice were placed in activity monitoring chambers for either $2 \mathrm{~h}$ (for terreic acid) or $4 \mathrm{~h}$ (for pergolide). Testing was carried out in automated monitors (Accuscan Instruments, Columbus, Ohio). Activity boxes were constructed of clear plastic chambers $(40 \times 40 \times 30 \mathrm{~cm})$ and activity was monitored from grids of $8 \times 8$ infrared beams affixed $6 \mathrm{~cm}$ above the test chamber floor and $2 \mathrm{~cm}$ above the floor. Software recorded beam breaks in 5 min epochs, which were summed across time periods of interest to calculate distance traveled during the assay.

\section{Behavioral Statistics}

We performed two-way ANOVAs (sex $\times$ dose) on data from the drinking studies and one-way ANOVAs on data from the locomotor activity assay using GraphPad Prism 7 and Systat v. 13. Where we identified a significant main effect of dose (in the absence of a significant sex $\times$ dose interaction), we followed up with one-way ANOVA and Tukey's post-hoc testing (corrected for multiple comparisons) to compare doses. Means \pm SEM are presented in the graphs of the behavioral data and $P<0.05$ was considered significant.

\section{RESULTS}

\section{Generation of the Input Query Signatures}

We performed a meta-analysis to identify differentially expressed genes that were dysregulated in ethanol-naive, male HDID-1 mice compared with $\mathrm{HS} / \mathrm{Npt}$ mice in each brain region (VS, PFC, AcbC, AcbSh, BNST, BLA, CeA, and VTA; see Materials and Methods). Genetic selection for drinking to intoxication was associated with global changes in gene expression between HDID-1 and HS/Npt mice, although these effects varied across brain regions. Only six genes were regulated across all eight brain areas (Pak1, Prcp, Ciapin1, Mrpl48, Klc1, and Atf4) (Supplementary Table S1). The finding that the effects of selection were highly brain region-specific reinforces the importance of submitting separate queries for each brain region. Two input signatures per brain region were constructed from the differentially expressed genes (HDID-1 vs HS/Npt mice) as described in the Materials and Methods.

\section{Identification and Prioritization of Drug Candidates}

We submitted a total of 32 queries to LINCS-L1000: two input signatures for each of the eight brain areas in two rowspaces (see Materials and Methods). Thus, each compound had 32 connectivity scores, 1 for each query (see Supplementary Table S2).

We prioritized compounds with highly negative connectivity scores across inputs (brain areas) to maximize the likelihood that the drug would reduce binge drinking. Multiple approaches were used to identify compounds with consistently negative connectivity scores (for details, see Materials and Methods; Figure 1 and Supplementary Figure S1). First, the resulting 32 scores/compound were summarized using the 75 th percentile score and then ranked from the least to greatest 75 th percentile score (Figures 1a and b). Quantiles provided a more useful descriptive statistic than averages, because the connectivity scores were not distributed according to any assumed distribution. For example, the connectivity scores could be normally distributed for one drug, but have a bimodal distribution for another drug. Compounds with a highly skewed distribution of connectivity scores were selected, as this would indicate highly negative scores for all inputs.

As an alternative approach, we used a threshold of -90 for each connectivity score and ranked drugs based on the total number of scores $\leqslant-90$ across the 32 queries (Figures $1 \mathrm{c}$ and d). Compounds were ranked according to the number of queries with connectivity scores $\leqslant-90$ (Figure 1c).

In addition, to increase the robustness of our approach, we used a third method based on a modified rank product score to prioritize compounds (Supplementary Figure S1) (Breitling et al, 2004; Fortney et al, 2015). For each input query, we ranked the compounds by connectivity score in increasing order (ie, rank 1 was assigned to the compound with the most negative connectivity score and rank 3021 was assigned to the compound with the most positive score). We calculated the product of connectivity score ranks for each of the 32 inputs and then prioritized compounds with the lowest modified rank product score.

Two compounds were consistently ranked in the top 15 from each of the 3 prioritization methods: terreic acid, a selective Bruton's tyrosine kinase (BTK) inhibitor and pergolide, a dopamine and serotonin receptor agonist used to treat Parkinson's disease (Figure 1e). Thus, we selected these candidates for in vivo testing.

\section{In vivo Testing of Drug Candidates}

Terreic acid. Acute administration of terreic acid produced a robust reduction in $20 \%$ ethanol intake (Figure $2 \mathrm{a}$; main effect of dose $\mathrm{F}(3,111)=8.9, P<0.0001)$. There was no significant effect of $\operatorname{sex}(\mathrm{F}(1,111)=2.8, \mathrm{P}>0.09)$ nor a sex $\times$ dose interaction $(\mathrm{F}(3,111)=1.2, P>0.29)$. To compare the effects of different terreic acid doses on ethanol intake, we followed up with a one-way ANOVA and Tukey's post-hoc test and found that $5,7.5$, and $15 \mathrm{mg} / \mathrm{kg}$ terreic acid reduced ethanol intake as compared with $0 \mathrm{mg} / \mathrm{kg}(P<0.01, P<0.01$, and $P<0.001$, respectively). 
a Compounds rank ordered by
$75^{\text {th }}$ percentile connectivity scor

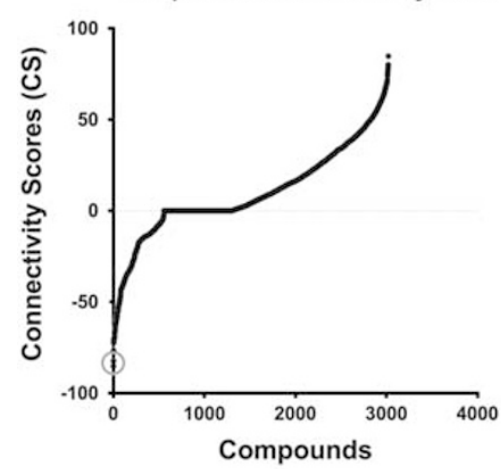

C Compounds rank ordered by significance threshold

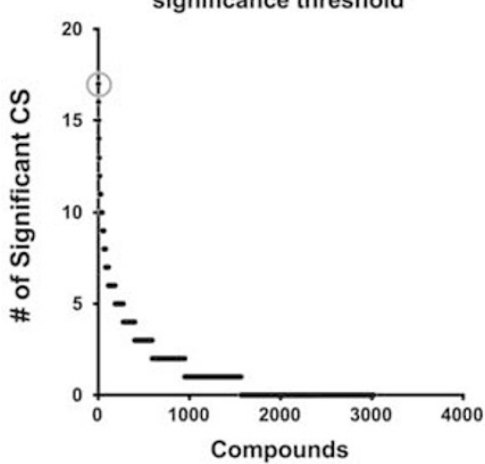

e

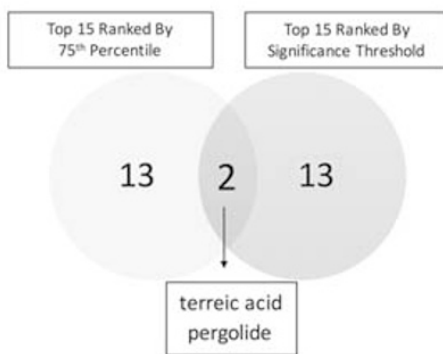

b

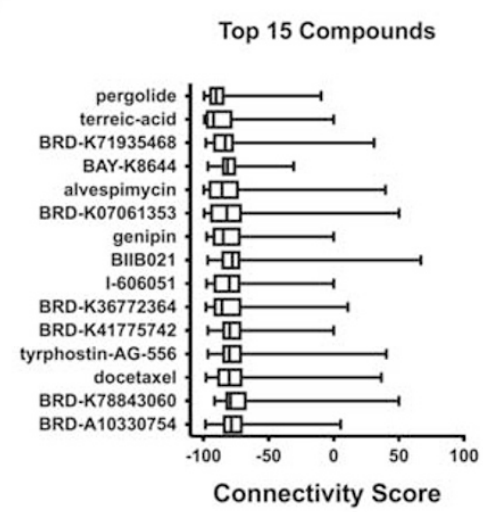

d

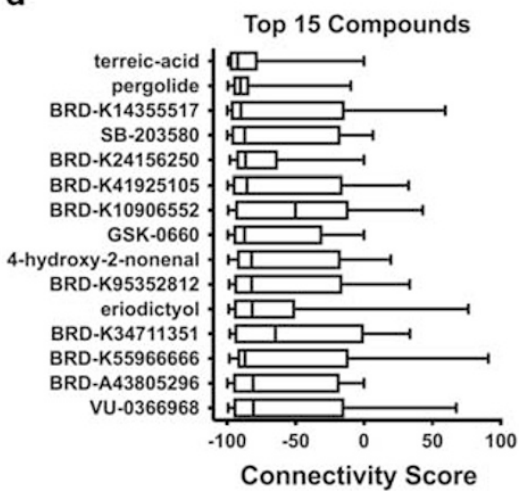

Figure I Prioritization of candidate compounds from the LINCS-LI 000 (Library of Integrated Network-Based Cellular Signatures) search results. Using the constructed input gene expression signatures (see Supplementary Table SI) with various algorithm parameter settings, a total of 32 input queries were submitted to LINCS using the sig_gutc tool via C3 (Compute Connectivity on the Cloud, see Materials and Methods). Queries represented the top differentially expressed genes or the differentially expressed landmark genes (those directly measured in the LINCS-LI000 database) between ethanol-naive, male High Drinking in the Dark (HDID-I), and HS/Npt (the founder population) mice across eight brain areas. The sig_gutc tool reports a summary connectivity score (aggregating across doses, time points, and cell lines) between the input signature and signatures of more than 3000 chemical compounds in the LINCS-LI 000 touchstone data set (comprised the most reproducible signatures for well-characterized compounds). Each compound had 32 scores (one for each input query). To identify compounds that consistently had negative scores across brain areas irrespective of the query parameter settings, we rank ordered compounds according to the 75th percentile score (out of the 32 scores) (a), and the number of significant connectivity scores (ie, $\leqslant-90$, see text) (out of the 32 scores) (c). The boxplots summarizing the connectivity scores across the 32 input queries for the top 15 compounds from each prioritization method are shown in b and d. Pergolide and terreic acid, circled in red in a and c, were the top 2 hits from both selection approaches (e). A full color version of this figure is available at the Neuropsychopharmacology journal online.

Acute administration of terreic acid resulted in a robust reduction in BALs achieved after ethanol drinking (Figure 2b; main effect of dose $\mathrm{F}(3,111)=7.4, P<0.001)$. There was no significant effect of sex $(\mathrm{F}(1,111)=0.1, P>0.74)$ nor a sex $\times$ dose interaction $(\mathrm{F}(3,111)=0.3, P>0.82)$. To compare the effects of different terreic acid doses on BAL, we followed up with a one-way ANOVA and Tukey's post-hoc test and found that 5, 7.5 , and $15 \mathrm{mg} / \mathrm{kg}$ terreic acid reduced BALs as compared with $0 \mathrm{mg} / \mathrm{kg}(P<0.05, P<0.05$, and $P<0.001$, respectively). Terreic acid reduced BALs below the level associated with binge drinking and intoxication in humans. Terreic acid also reduced water intake (Figure $2 c$; main effect of dose: $\mathrm{F}(3,111)=9.9, \mathrm{P}<0.0001$ ). We observed a main effect of sex $(\mathrm{F}>\mathrm{M} ; \mathrm{F}(1,111)=4.5$, $P<0.05)$, but not a sex $\times$ dose interaction $(F(3,111)=0.7$, $P>0.56$ ). To compare the effects of different terreic acid doses on water intake, we followed up with a one-way ANOVA and Tukey's post-hoc test and found that $15 \mathrm{mg} / \mathrm{kg}$ terreic acid reduced water intake as compared with 0,5 , and $7.5 \mathrm{mg} / \mathrm{kg}$ $(P<0.0001, P<0.001$, and $P<0.01$, respectively). To determine whether terreic acid might reduce fluid intake in a nonselective 

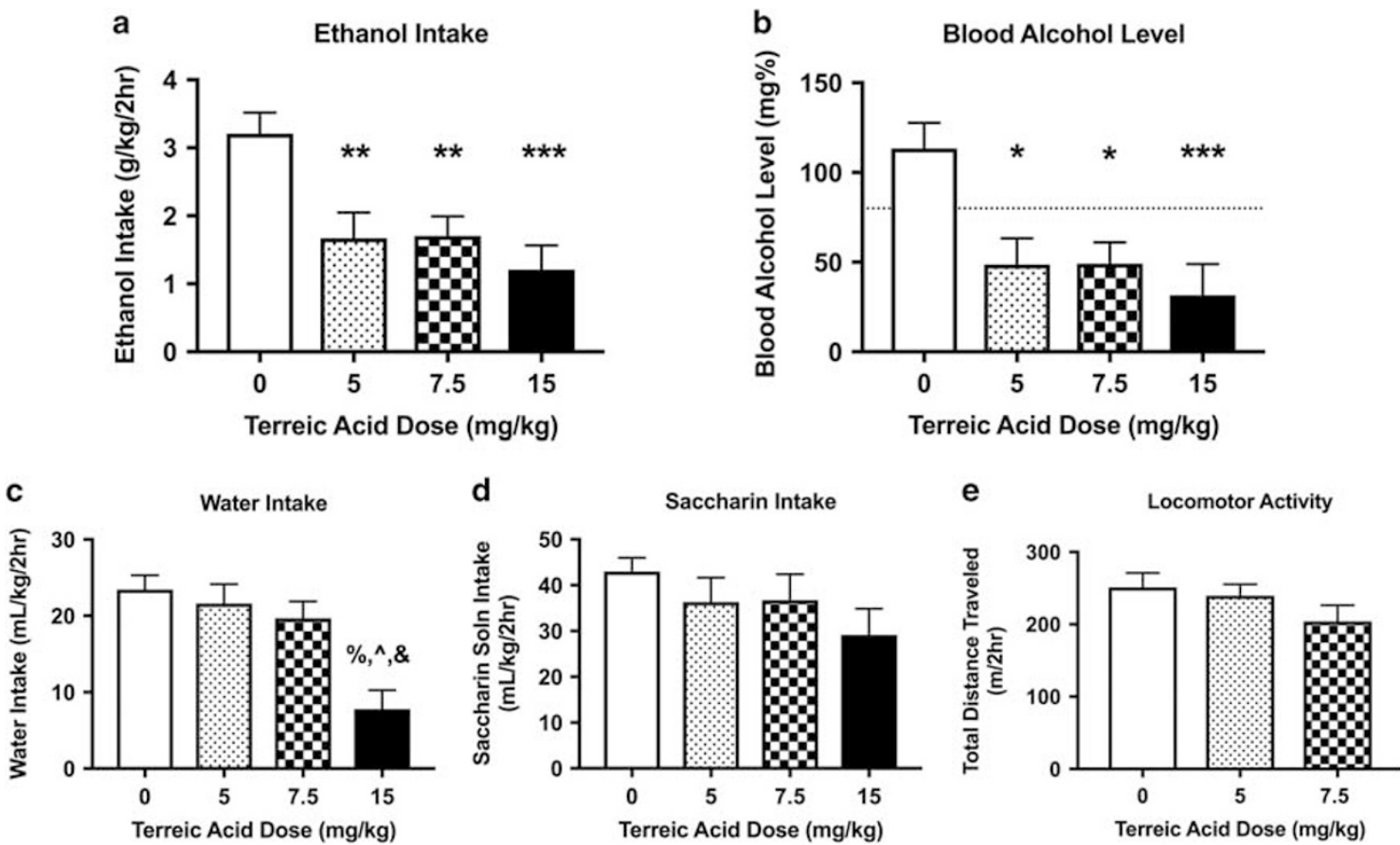

Figure 2 Effects of the candidate drug, terreic acid, on binge drinking in High Drinking in the Dark (HDID-I) mice. Terreic acid significantly reduced bingelike ethanol (20\%) intake (a) and blood alcohol levels (BALs) (b). NIAAA defines binge drinking as a pattern of drinking that produces BALs of 80 mg\%, designated in b by a dashed line (at $y=80$ ). Although the highest dose of terreic acid tested reduced water intake (c), none of the doses tested significantly reduced intake of saccharin $(8.5 \mathrm{mM})(\mathrm{d})$. Furthermore, doses of terreic acid that reduced ethanol intake and BAL did not alter locomotor activity (e). Results of Tukey's post-hoc analysis are indicated above the SEM bars. In a and $b, * P<0.05$, $* * P<0.0$ I, and $* * * P<0.00$ I vs the 0 -dose groups and in $c \%=P<0.000$ I for 15 vs $0 \mathrm{mg} / \mathrm{kg}, \wedge P<0.00$ I for 15 vs $5 \mathrm{mg} / \mathrm{kg}$, and $\& P<0.01$ for 15 vs $7.5 \mathrm{mg} / \mathrm{kg}$ terreic acid. Values represent mean \pm SEM (Drinking assays: $n=12-24 / \mathrm{sex}$ ) dose; data shown are collapsed on sex, thus each bar represents 24-47 mice; Activity assay: $n=7-8 /$ sex/dose).
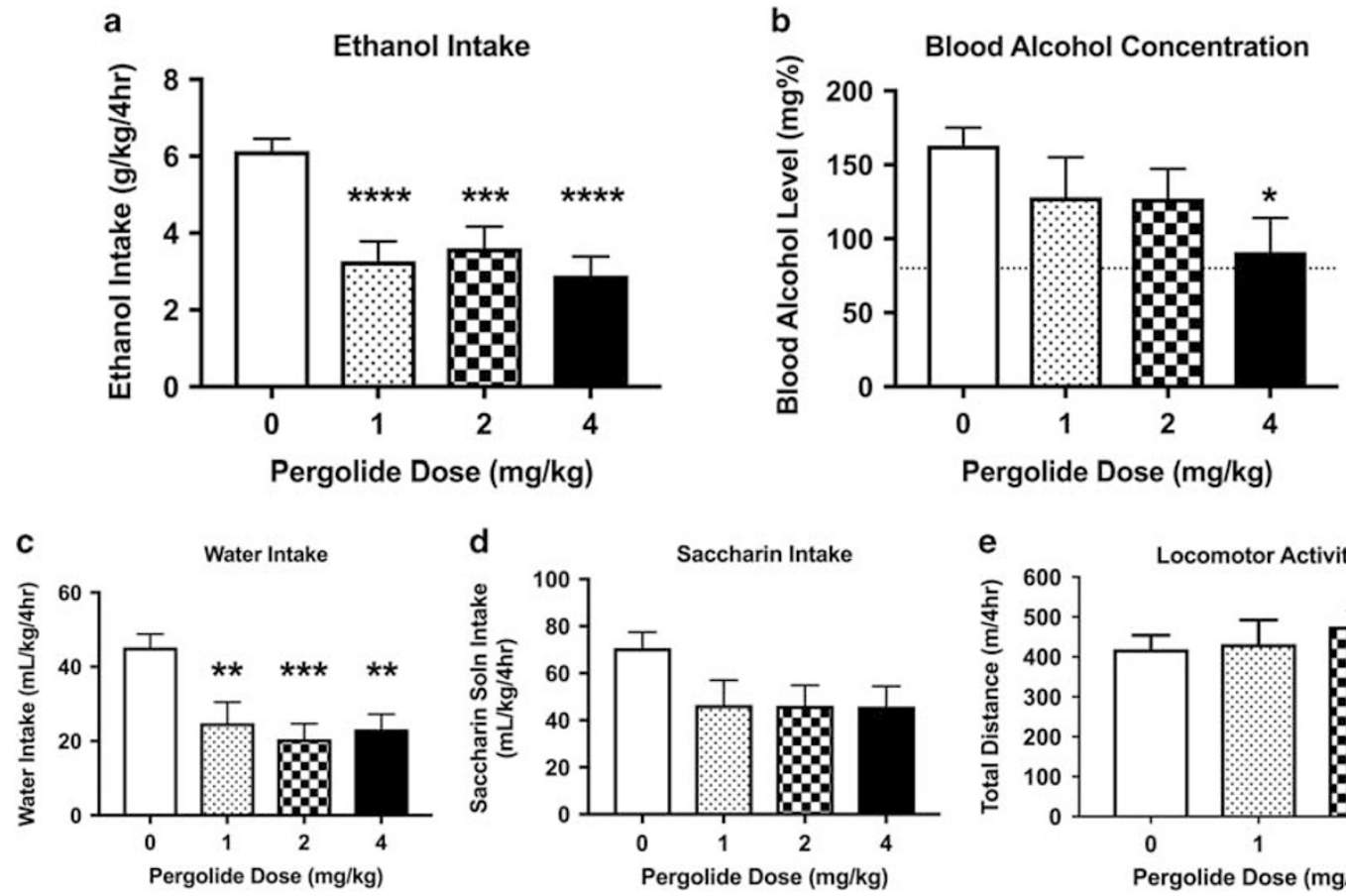

Figure 3 Effects of the candidate drug, pergolide, on binge drinking in High Drinking in the Dark (HDID-I) mice. Pergolide significantly reduced binge-like ethanol (20\%) intake (a) and blood alcohol levels (BALs) (b). Pergolide also significantly reduced water (c) and 8.5 mM saccharin (d) intake but did not alter locomotor activity (e). Results of Tukey's post-hoc analysis are indicated above the SEM bars $(* P<0.05, * * P<0.0$ I, $* * * *<<0.00$ I, ***** $P<0.000$ I vs the 0 -dose groups). Values represent mean \pm SEM (Drinking assays: $n=12-24 /$ sex/dose; data shown are collapsed on sex, thus each bar represents $24-47$ mice; Activity assay: $n=7-8 /$ sex/dose). 
manner or produce general malaise, we assayed its effects on saccharin consumption in HDID-1 mice. Terreic acid did not alter the intake of $8.5 \mathrm{mM}$ saccharin (Figure $2 \mathrm{~d} ; \mathrm{F}(3,107)=1.7$, $P>0.16)$. There was a significant effect of $\operatorname{sex}(\mathrm{F}>\mathrm{M} ; \mathrm{F}$ $(1,107)=6.9, P<0.01)$, but no sex $\times$ dose interaction $(\mathrm{F}(3$, $107)=0.7, P>0.52)$. Lastly, we assessed the effects of terreic acid on locomotor activity (at the same time of day that the drinking studies were carried out). Administration of 5 or $7.5 \mathrm{mg} / \mathrm{kg}$ terreic acid did not alter locomotor activity (Figure 2e; $\mathrm{F}(2,44)=1.6, P>0.21)$. Therefore, reductions in ethanol intake cannot be explained by terreic acid-induced changes in malaise or by a general sedative effect of the drug on locomotor activity.

Pergolide. Acute administration of pergolide produced a robust reduction in $20 \%$ ethanol intake (Figure 3a; main effect of dose $\mathrm{F}(3,109)=18.7, P<0.0001)$. There was a significant effect of sex $(\mathrm{F}>\mathrm{M} ; \mathrm{F}(1,109)=2.8, P<0.09)$ but no sex $\times$ dose interaction $(\mathrm{F}(3,109)=0.8, P>0.47)$. To compare the effects of different pergolide doses on ethanol intake, we followed up with a one-way ANOVA and Tukey's post-hoc test and found that 1, 2, and $4 \mathrm{mg} / \mathrm{kg}$ pergolide reduced ethanol intake as compared with $0 \mathrm{mg} / \mathrm{kg}$ $(P<0.0001, P<0.001$, and $P<0.0001$, respectively). Acute administration of pergolide resulted in reduced BALs (Figure 3b; main effect of dose $\mathrm{F}(3,109)=3.2, P<0.05)$. There was a significant effect of sex $(F>M ; F(1,109)=9.9$, $P<0.01)$, but no sex $\times$ dose interaction $(\mathrm{F}(3,109)=1.7$, $P>0.16)$. To compare the effects of different pergolide doses on BAL, we followed up with a one-way ANOVA and Tukey's post-hoc test and found that $4 \mathrm{mg} / \mathrm{kg}$ pergolide reduced $\mathrm{BAL}$ as compared with $0 \mathrm{mg} / \mathrm{kg}(P<0.05)$. Pergolide also reduced water intake (Figure $3 \mathrm{c}$; main effect of dose: $\mathrm{F}$ $(3,109)=8.3, P<0.0001)$. We did not observe a significant main effect of sex $(F(1,109)=3.8, P>0.05)$, nor did we observe a sex $\mathrm{x}$ dose interaction $(\mathrm{F}(3,109)=1.1, P>0.36)$. To compare the effects of different pergolide doses on water intake, we followed up with a one-way ANOVA and Tukey's post-hoc test and found that 1,2, and $4 \mathrm{mg} / \mathrm{kg}$ pergolide reduced water intake as compared with vehicle $(P<0.01$, $P<0.001$, and $P<0.01$, respectively). To determine whether pergolide might reduce fluid intake in a nonselective manner or produce general malaise, we assayed its effects on saccharin consumption in HDID-1 mice. Pergolide also resulted in reductions in the intake of $8.5 \mathrm{mM}$ saccharin (Figure 3d; $\mathrm{F}(3,107)=3.1, P<0.05)$. There was a significant effect of sex $(\mathrm{F}>\mathrm{M} ; \mathrm{F}(1,107)=12.8, P<0.001)$, but no sex $\times$ dose interaction $(\mathrm{F}(3,107)=0.5, P>0.69)$. We did not identify effects of specific doses on saccharin intake (via oneway ANOVA and Tukey's post-hoc tests). Lastly, we assessed the effects of terreic acid on locomotor activity (at the same time of day that the drinking studies were carried out). Administration of 1 or $2 \mathrm{mg} / \mathrm{kg}$ terreic acid did not alter locomotor activity (Figure $3 \mathrm{e} ; \mathrm{F}(2,44)=0.4, P>0.66$ ). Therefore, reductions in fluid intake cannot be explained by pergolide-induced changes in locomotor activity.

\section{DISCUSSION}

\section{Drug Development and Repurposing}

The classic pharmacological approach of designing compounds for a single molecular target has largely been unsuccessful for the treatment of central nervous system diseases (Hutson et al, 2017). Of the 45 drugs approved by the FDA in 2015, only 3 were indicated for psychiatric disorders (Breen et al, 2016). The last (and only the third) FDA-approved treatment for AUD was acamprosate in 2004. Clearly, new approaches are required to advance treatment options for AUD and other psychiatric illnesses, which show extensive comorbidity (Fein, 2015). Our study highlights the utility of emerging computational approaches to prioritize compounds that may target molecular profiles associated with binge drinking, and potentially 'drug' the altered gene networks. We provide the first evidence that brain gene expression data can be combined with the LINCS-L1000 database to identify new compounds that reduce drinking in a genetic animal model of AUD risk.

The cost and time investment for drug discovery roughly doubles every 9 years (Nosengo, 2016). Therefore, drug repurposing (adapting the use of existing compounds to target other symptoms or diseases) is an expedient and costefficient pathway to FDA approval given that the drug profile data have already been established (eg, pharmacodynamics, bioavailability, kinetics, interactions, toxicity, and side effects). The LINCS-L1000 database contains many FDAapproved drugs, making the approaches outlined here an excellent pathway for repurposing efforts.

As an initial proof-of-concept, we chose the top 2 ranked compounds to test in HDID-1 mice, irrespective of their future potential use in humans. There are additional safety and efficacy considerations that would need to be evaluated for terreic acid or pergolide to become repurposed candidates for AUD treatment in humans. Regardless of whether terreic acid and/or pergolide are pursued for AUD treatment, our study highlights the ability of our novel approach to rapidly identify novel drugs/targets that reduce alcohol intake and attests to its potential utility to predict candidates for AUD treatment. Future studies will explore alternative LINCS-L1000 queries using existing gene expression data sets from humans and other animal models of AUD to continue refining our gene network approach and focus on FDA-approved compounds within LINCS-L1000.

\section{Using Omics Data from Animal Models to Select Candidate Drugs}

In this study, we utilized HDID-1 mice as a genetic model of high intensity, binge-like drinking, and as a predictive model for drug discovery. There are numerous advantages of this model (Barkley-Levenson and Crabbe, 2014). For example, $>90 \%$ of HDID-1 mice achieve pharmacologically relevant BALs after binge drinking. They also show behavioral impairment after binge-like drinking, escalate their intake in response to the development of dependence (relapse-like drinking), and exhibit withdrawal after a single binge session. Importantly, genetic selection has not altered their preference for other tastants or their ability to metabolize alcohol (Crabbe et al, 2011). Overall, the value of individual animal models can be attributed to their construct, face, and predictive validity. HDID-1 mice have been shown to have valuable construct and face validity, fulfilling many criteria proposed for an animal model of excessive alcohol consumption (reviewed in Barkley-Levenson and Crabbe, 2014). Our study provides evidence that the HDID-1 
model also offers predictive validity for successful drug identification.

Genetic selection for drinking to intoxication was associated with varying transcriptome changes across different brain regions. As the brain areas that are most critical for the specific binge drinking phenotype are not well defined and because candidate drugs are administered systemically and can reach many tissues, it may be important to incorporate data from several brain regions important for addictive behavior (Koob and Mason, 2016; Ozburn et al, 2015). We thus included different gene expression datasets that profiled the transcriptomic state across eight brain regions which helped to mitigate technological and biological biases (similar to other approaches (Fortney et al, 2015; Liu et al, 2015)). Instead of collapsing the available gene expression datasets at the gene level and identifying a single molecular signature, we ran each data set as a separate LINCS-L1000 query to prioritize drugs with the potential to target the transcriptome in many brain areas, which has been shown to produce candidate compounds more reliably (Fortney et al, 2015).

\section{Rigor of Bioinformatics Approach}

Successful use of CMap and LINCS-L1000 for drug discovery and repurposing requires multiple approaches, which may be different depending on the tissue type, disease, and a host of other specific factors. However, a systematic evaluation of the impact of parameter settings had not been tested previously. Here we used multiple expression data sets, algorithm parameter settings, and methods for prioritizing compounds to increase the rigor and robustness of the results and the ability to identify an effective drug candidate. Another merit of our approach, and one that was likely critical to our success, was focusing on top candidates identified by all approaches across methodological choices.

The LINCS-L1000 reference library represents transcriptional responses to compounds in different human cell types from different tissue types (including two cell lines derived from brain). The mouse brain tissue used to generate the input signatures associated with binge drinking is also heterogeneous (representing a diversity of brain cell types), and yet, when tested in vivo, the drug candidates were effective when delivered systemically (reaching many cell types). This indicates that the targeted signatures likely affect widespread biological responses and provides compelling evidence that the LINCS-L1000 library can be applied to many different diseases, even when there is not complete concordance in cell and tissue types. Cross-species and crosscell-type generalizability is consistent with other successful applications of CMap/LINCS-L1000 (Kidd et al, 2016; Liu et al, 2015).

\section{Candidate Drugs for In Vivo Testing}

Low doses of terreic acid and pergolide robustly decreased alcohol intake in HDID-1 mice and reduced BALs. We note that low doses of terreic acid appeared to reduce binge-like ethanol drinking selectively, as we saw decreased water intake only at the highest dose tested. In addition, we observed no reduction in saccharin intake or locomotor activity at any of the doses tested for terreic acid. The

behavioral profile of pergolide appeared to be less selective for binge-like ethanol drinking, where we observed decreased water intake for all doses tested, and reduced saccharin intake. However, since pergolide did not alter locomotor activity, reductions in fluid intake cannot be explained by pergolide-induced sedation or malaise. It is well known that dopamine is essential for normal locomotor, feeding and drinking behavior (Myers, 1969; Szczypka et al, 1999; Zabik et al, 1993). Most relevant to the current study, pergolide $(2 \mathrm{mg} / \mathrm{kg}$; ip) increased water intake in rats during singlebottle, limited access conditions (closely mirroring the current study) (Zabik et al, 1993). It is possible that the effects of pergolide in mouse are opposite of those in rat. However, whether all dopamine agonists increased or decreased water intake is inconsistent across studies and seemed to depend on initial dopamine levels, route of administration, time of day, and species, to name a few experimental factors. We do not think the reduced water consumption we report here is an indication that these compounds cause general malaise. Animals are not physiologically challenged by the DID drinking test, which does not involve general fluid deprivation (Toth and Gardiner, 2000). All animals appeared normal, and pergolide, which had the larger effect on water intake of the two compounds, is used clinically for Parkinson's in Europe and does not decrease water consumption or cause malaise in those patients. Furthermore, short-lasting reductions in water intake are quite common in pre-clinical testing following intraperitoneal drug administration.

The LINCS-L1000 input signature is designed to capture the components of the expression signal that are most relevant to the disease state, and does not include housekeeping or other non-disease-related genes. However, determining which genes is a complex process, representing a dynamic, evolving area of research. Here we identified the top differentially expressed known genes and differentially expressed landmark genes to construct HDID signatures that reflect the genetic selection for intoxication after binge-like alcohol drinking (Supplementary Table S1). Future experiments will integrate the transcriptomic signatures of drugs and diseases at the gene-set level (ie, gene network/modular level) as an alternative approach that might represent a more refined input signature to identify compounds with greater selectivity for ethanol consumption, and has shown some success in CMap/LINCS-L1000 applications (Chung et al, 2015; Siavelis et al, 2016).

The identification of terreic acid, a BTK inhibitor with antibiotic, anti-inflammatory, and anti-oxidative activity, is especially noteworthy since this compound would not have been predicted based on prior alcohol research. To the best of our knowledge, BTK has not been implicated in any actions of alcohol. BTK is expressed in the periphery, as well as in microglia (Zhang et al, 2014). Pharmacological inhibition of BTK is known to reduce inflammation and pro-inflammatory cytokine expression. Specifically, BTK inhibition reduces immune hypersensitivity responses and arthritis in rodents (Xu et al, 2012) and suppresses infarct volume growth and neurological damage in a brain ischemia model in mice (Ito et al, 2015). Pergolide also has anti-inflammatory actions (Bendele et al, 1991). Recent work has shown that increasing levels of pro-inflammatory cytokines increases alcohol drinking and reducing these levels can reduce drinking in C57BL/6 
mice (see Crews et al, 2017 for review). Genipin, the aglycon of geniposide found in gardenia fruit, and alvespimycin, a HSP90 inhibitor, were also highly ranked among all three prioritization methods. Both have been reported to have antiinflammatory effects as well (Madrigal-Matute et al, 2010; Nam et al, 2010). Another top ranking compound identified in our analysis was BRD-K14355517 (Pubchem cid: 3989078), a neuropeptide $\mathrm{S}$ antagonist. Interestingly, a similar neuropeptide S antagonist, NCGC00185684, reduced alcohol consumption in rats (Thorsell et al, 2013). Neuropeptides are biochemical mediators in neuroimmune crosstalk and can counterbalance inflammatory responses (Delgado and Ganea, 2008). Although not initially selected for in vivo testing, these compounds could also have potential to decrease binge drinking in HDID-1 mice. Overall, our findings support a key role for drugs that target inflammatory/immune pathways as potential therapeutics to reduce alcohol drinking, in agreement with neuroimmune evidence obtained from other mouse models of alcohol consumption and human alcoholics. Focusing repurposing on drugs that target neuroimmune signaling may be a promising area for AUD drug discovery.

In summary, our proof-of-concept findings highlight the power of systems genomics approaches to successfully predict compounds (and identify relevant gene targets) with translational potential to reduce drinking. We identified novel compounds (eg, a BTK inhibitor, HSP90 inhibitor, neuropeptide $\mathrm{S}$ antagonist, and dopamine/serotonin agonist), many with anti-inflammatory properties, providing further support for a neuroimmune mechanism of excessive alcohol drinking. We have shown that the HDID mouse model, together with multiple computational methods, offer predictive validity for drug discovery or repurposing to reduce binge-like drinking. These novel approaches are part of a dynamic, evolving area of research that promises to uncover and expedite targeted drug treatments for AUD and other psychiatric diseases.

\section{FUNDING AND DISCLOSURE}

These studies are a part of the Integrative Neuroscience Initiative on Alcoholism-Neuroimmune, an NIAAAsponsored consortium effort (U01 AA020926 (Parent Admin Supp) to RDM; U01 AA13519 to JCC and ARO). Additional support: NIH (P01 AA020683 to RDM; R01 AA012404 to RAH and RDM; R24 AA020245 to JCC, F31 AA024332 to LF, AA017234 to IP), US Department of Veterans Affairs Awards (IK2 BX002488 to ARO, 101BX000313 to JCC), BBRF NARSAD Young Investigator Award to ARO, Andrews Genomics Fund to ARO, and $\mathrm{NIH}$ Center grant AA10760 to Tamara Phillips (PI; JCC, CoI). The authors declare no conflict of interest.

\section{ACKNOWLEDGMENTS}

We thank Stephanie Spence, Evan Firsick, Jason Schlumbohm, Lawrence Huang, and Wyatt Hack for technical assistance; Deborah Finn for help with some blood alcohol analyses; and Jody Mayfield for editorial assistance and helpful comments. Finally, we dedicate this to the late Matt Reilly, without whom this work would simply not exist.

\section{REFERENCES}

Barkley-Levenson AM, Crabbe JC (2014). High drinking in the dark mice: a genetic model of drinking to intoxication. Alcohol 48: 217-223.

Bendele AM, Spaethe SM, Benslay DN, Bryant HU (1991). Antiinflammatory activity of pergolide, a dopamine receptor agonist. J Pharmacol Exp Ther 259: 169-175.

Breen G, Li Q, Roth BL, O'Donnell P, Didriksen M, Dolmetsch R et al (2016). Translating genome-wide association findings into new therapeutics for psychiatry. Nat Neurosci 19: 1392-1396.

Breitling R, Armengaud P, Amtmann A, Herzyk P (2004). Rank products: a simple, yet powerful, new method to detect differentially regulated genes in replicated microarray experiments. FEBS Lett 573: 83-92.

Carlson M (2016). hgu133a.db: Affymetrix Human Genome U133 Set annotation data (chip hgu133a). $R$ package version 323.

Chen B, Butte AJ (2016). Leveraging big data to transform target selection and drug discovery. Clin Pharmacol Ther 99: 285-297.

Chen B, Ma L, Paik H, Sirota M, Wei W, Chua MS et al (2017). Reversal of cancer gene expression correlates with drug efficacy and reveals therapeutic targets. Nat Commun 8: 16022.

Chung FH, Jin ZH, Hsu TT, Hsu CL, Liu HC, Lee HC (2015). Gene-Set Local Hierarchical Clustering (GSLHC)-a gene set-based approach for characterizing bioactive compounds in terms of biological functional groups. PLOS ONE 10: e0139889.

Crabbe JC, Metten P, Belknap JK, Spence SE, Cameron AJ, Schlumbohm JP et al (2014). Progress in a replicated selection for elevated blood ethanol concentrations in HDID mice. Genes Brain Behav 13: 236-246.

Crabbe JC, Metten P, Rhodes JS, Yu CH, Brown LL, Phillips TJ et al (2009). A line of mice selected for high blood ethanol concentrations shows drinking in the dark to intoxication. Biol Psychiatry 65: 662-670.

Crabbe JC, Spence SE, Brown LL, Metten P (2011). Alcohol preference drinking in a mouse line selectively bred for high drinking in the dark. Alcohol 45: 427-440.

Crews FT, Lawrimore CJ, Walter TJ, Coleman LG Jr (2017). The role of neuroimmune signaling in alcoholism. Neuropharmacology 122: 56-73.

Delgado M, Ganea D (2008). Anti-inflammatory neuropeptides: a new class of endogenous immunoregulatory agents. Brain Behav Immun 22: 1146-1151.

Du P, Kibbe WA, Lin SM (2008). lumi: a pipeline for processing Illumina microarray. Bioinformatics 24: 1547-1548.

Esser MB, Hedden SL, Kanny D, Brewer RD, Gfroerer JC, Naimi TS (2014). Prevalence of alcohol dependence among US adult drinkers, 2009-2011. Prev Chronic Dis 11: E206.

Fein G (2015). Psychiatric comorbidity in alcohol dependence. Neuropsychol Rev 25: 456-475.

Finn DA, Snelling C, Fretwell AM, Tanchuck MA, Underwood L, Cole $\mathrm{M}$ et al (2007). Increased drinking during withdrawal from intermittent ethanol exposure is blocked by the CRF receptor antagonist D-Phe-CRF(12-41). Alcohol Clin Exp Res 31: 939-949.

Fortney K, Griesman J, Kotlyar M, Pastrello C, Angeli M, SoundTsao $\mathrm{M}$ et al (2015). Prioritizing therapeutics for lung cancer: an integrative meta-analysis of cancer gene signatures and chemogenomic data. PLoS Computat Biol 11: e1004068.

Fowler KD, Funt JM, Artyomov MN, Zeskind B, Kolitz SE, Towfic F (2015). Leveraging existing data sets to generate new insights into Alzheimer's disease biology in specific patient subsets. Sci Rep 5: 14324.

Gao L, Zhao G, Fang JS, Yuan TY, Liu AL, Du GH (2014). Discovery of the neuroprotective effects of alvespimycin by computational prioritization of potential anti-Parkinson agents. FEBS J 281: 1110-1122.

Han H, Yang Y, Olesen SH, Becker A, Betzi S, Schonbrunn E (2010). The fungal product terreic acid is a covalent inhibitor of the 
bacterial cell wall biosynthetic enzyme UDP-N-acetylglucosamine 1-carboxyvinyltransferase (MurA). Biochemistry 49: 4276-4282.

Hutson PH, Clark JA, Cross AJ (2017). CNS target identification and validation: avoiding the valley of death or naive optimism? Annu Rev Pharmacol Toxicol 57: 171-187.

Iancu OD, Oberbeck D, Darakjian P, Metten P, McWeeney S, Crabbe JC et al (2013). Selection for drinking in the dark alters brain gene coexpression networks. Alcohol Clin Exp Res 37: 1295-1303.

Iorio F, Rittman T, Ge H, Menden M, Saez-Rodriguez J (2013). Transcriptional data: a new gateway to drug repositioning? Drug Discov Today 18: 350-357.

Ito $\mathrm{M}$, Shichita T, Okada M, Komine R, Noguchi Y, Yoshimura A et al (2015). Bruton's tyrosine kinase is essential for NLRP3 inflammasome activation and contributes to ischaemic brain injury. Nat Commun 6: 7360.

Kawakami Y, Hartman SE, Kinoshita E, Suzuki H, Kitaura J, Yao L et al (1999). Terreic acid, a quinone epoxide inhibitor of Bruton's tyrosine kinase. Proc Natl Acad Sci USA 96: 2227-2232.

Kawakami Y, Yumoto K, Kawakami T (2007). An improved mouse model of atopic dermatitis and suppression of skin lesions by an inhibitor of Tec family kinases. Allergol Int 56: 403-409.

Kidd BA, Wroblewska A, Boland MR, Agudo J, Merad M, Tatonetti NP et al (2016). Mapping the effects of drugs on the immune system. Nat Biotechnol 34: 47-54.

Koob GF, Mason BJ (2016). Existing and future drugs for the treatment of the dark side of addiction. Annu Rev Pharmacol Toxicol 56: 299-322.

Lamb J, Crawford ED, Peck D, Modell JW, Blat IC, Wrobel MJ et al (2006). The Connectivity Map: using gene-expression signatures to connect small molecules, genes, and disease. Science 313: 1929-1935.

Lipponen A, Paananen J, Puhakka N, Pitkanen A (2016). Analysis of post-traumatic brain injury gene expression signature reveals tubulins, Nfe212, Nfkb, Cd44, and S100a4 as treatment targets. Sci Rep 6: 31570.

Liu J, Lee J, Salazar Hernandez MA, Mazitschek R, Ozcan U (2015). Treatment of obesity with celastrol. Cell 161: 999-1011.

Madrigal-Matute J, Lopez-Franco O, Blanco-Colio LM, Munoz-Garcia B, Ramos-Mozo P, Ortega L et al (2010). Heat shock protein 90 inhibitors attenuate inflammatory responses in atherosclerosis. Cardiovasc Res 86: 330-337.

Martin GE, Bendesky RJ (1984). Mouse locomotor activity: an in vivo test for dopamine autoreceptor activation. J Pharmacol Exp Ther 229: 706-711.

Mirza N, Sills GJ, Pirmohamed M, Marson AG (2017). Identifying new antiepileptic drugs through genomics-based drug repurposing. Hum Mol Genet 26: 527-537.

Myers RD (1969). Chemical mechanisms in the hypothalamus mediating eating and drinking in the monkey. Ann N Y Acad Sci 157: 918-933.

Nakagawa S, Wei L, Song WM, Higashi T, Ghoshal S, Kim RS et al (2016). Molecular liver cancer prevention in cirrhosis by organ transcriptome analysis and lysophosphatidic acid pathway inhibition. Cancer Cell 30: 879-890.

Nam KN, Choi YS, Jung HJ, Park GH, Park JM, Moon SK et al (2010). Genipin inhibits the inflammatory response of rat brain microglial cells. Int Immunopharmacol 10: 493-499.
Nosengo N (2016). Can you teach old drugs new tricks? Nature 534: 314-316.

Olesen SH, Ingles DJ, Yang Y, Schonbrunn E (2014). Differential antibacterial properties of the MurA inhibitors terreic acid and fosfomycin. J Basic Microbiol 54: 322-326.

Ozburn AR, Janowsky AJ, Crabbe JC (2015). Commonalities and distinctions among mechanisms of addiction to alcohol and other drugs. Alcohol Clin Exp Res 39: 1863-1877.

Ralph-Williams RJ, Lehmann-Masten V, Geyer MA (2003). Dopamine D1 rather than D2 receptor agonists disrupt prepulse inhibition of startle in mice. Neuropsychopharmacology 28: 108-118.

Ritchie ME, Phipson B, Wu D, Hu Y, Law CW, Shi W et al (2015). limma powers differential expression analyses for RNA-sequencing and microarray studies. Nucleic Acids Res 43: e47.

Siavelis JC, Bourdakou MM, Athanasiadis EI, Spyrou GM, Nikita KS (2016). Bioinformatics methods in drug repurposing for Alzheimer's disease. Brief Bioinform 17: 322-335.

Smalley JL, Breda C, Mason RP, Kooner G, Luthi-Carter R, Gant TW et al (2016). Connectivity mapping uncovers small molecules that modulate neurodegeneration in Huntington's disease models. J Mol Med 94: 235-245.

So HC, Chau CK, Chiu WT, Ho KS, Lo CP, Yim SH et al (2017). Analysis of genome-wide association data highlights candidates for drug repositioning in psychiatry. Nat Neurosci 20: 1342-1349.

Subramanian A, Narayan R, Corsello SM, Peck DD, Natoli TE, Lu X et al (2017). A next generation connectivity map: L1000 platform and the first 1,000,000 profiles. Cell 171(6): 1437-1452 e1417.

Szczypka MS, Rainey MA, Kim DS, Alaynick WA, Marck BT, Matsumoto AM et al (1999). Feeding behavior in dopaminedeficient mice. Proc Natl Acad Sci USA 96: 12138-12143.

Thorsell A, Tapocik JD, Liu K, Zook M, Bell L, Flanigan M et al (2013). A novel brain penetrant NPS receptor antagonist, NCGC00185684, blocks alcohol-induced ERK-phosphorylation in the central amygdala and decreases operant alcohol selfadministration in rats. $J$ Neurosci 33: 10132-10142.

Toth LA, Gardiner TW (2000). Food and water restriction protocols: physiological and behavioral considerations. Contemp Top Lab Anim Sci 39: 9-17.

Wagner A, Cohen N, Kelder T, Amit U, Liebman E, Steinberg DM et al (2015). Drugs that reverse disease transcriptomic signatures are more effective in a mouse model of dyslipidemia. Mol Syst Biol 11: 791.

Xu D, Kim Y, Postelnek J, Vu MD, Hu DQ, Liao C et al (2012). RN486, a selective Bruton's tyrosine kinase inhibitor, abrogates immune hypersensitivity responses and arthritis in rodents. $J$ Pharmacol Exp Ther 341: 90-103.

Yamamoto H, Moriyama K, Jinnouchi H, Yagishita K (1980). Studies on terreic acid. Jpn J Antibiot 33: 320-328.

Zabik JE, Sprague JE, Odio M (1993). Interactive dopaminergic and noradrenergic systems in the regulation of thirst in the rat. Physiol Behav 54: 29-33.

Zhang Y, Chen K, Sloan SA, Bennett ML, Scholze AR, O'Keeffe S et al (2014). An RNA-sequencing transcriptome and splicing database of glia, neurons, and vascular cells of the cerebral cortex. J Neurosci 34: 11929-11947.

Supplementary Information accompanies the paper on the Neuropsychopharmacology website (http://www.nature.com/npp) 